Notfall Rettungsmed $2022 \cdot 25: 541-551$ https://doi.org/10.1007/s10049-021-00949-y Angenommen: 6. September 2021 Online publiziert: 17. November 2021 ๑) Der/die Autor(en) 2021

\section{Sepsis in der prähospitalen Notfallmedizin}

\section{Was können prähospital Tätige aus der Surviving Sepsis Campaign und S3-Leitlinie ziehen?}

Manuel Obermaier · Markus A. Weigand · Erik Popp · Florian Uhle

Klinik für Anästhesiologie, Universitätsklinikum Heidelberg, Heidelberg, Deutschland

\title{
Zusammenfassung
}

Hintergrund: Die Sepsis ist eine notfallmedizinische Herausforderung - denn diese lebensbedrohliche Organdysfunktion, verursacht durch eine dysregulierte Wirtsantwort auf eine Infektion, präsentiert sich in vielfältiger Ausprägung und wird deshalb häufig erst zu spät erkannt.

Fragestellung: Die kürzlich publizierten „surviving sepsis campaign“-Guidelines und die deutsche S3-Leitlinie geben Empfehlungen zur Diagnostik und Therapie der Sepsis im intrahospitalen bzw. intensivmedizinischen Setting, gehen jedoch nicht explizit auf den Bereich der prähospitalen Notfallmedizin ein. Ziel der Arbeit ist es, die Evidenzlage im Hinblick auf die prähospitale Versorgung von Patienten mit Verdacht auf Sepsis herauszuarbeiten und daraus Handlungsoptionen für den Notarzt- und Rettungsdienst abzuleiten.

Diskussion: Die Therapie der Sepsis und des septischen Schocks wird in Bündeln zusammengefasst, wobei das erste idealerweise innerhalb der ersten Stunde abgeschlossen sein soll - analog zum Konzept der "golden hour" bei anderen notfallmedizinischen Entitäten wie der Traumaversorgung. Die prähospitale Therapie fokussiert sich auf die Sicherung der Vitalparameter gemäß ABCDE-Schema, wobei insbesondere der Volumentherapie ein hoher Stellenwert zukommt. Die weiteren Maßnahmen des „1 $\mathrm{h}$ bundle“, wie Laktatmessung, Gewinnung mikrobiologischer Proben und Beginn einer antiinfektiven Therapie, sind regelhaft erst in der Klinik möglich. Ziel ist eine schnellstmögliche Fokussanierung, wofür die Auswahl und Vorabinformation einer geeigneten Zielklinik zur Initiierung und Bahnung der weiteren klinischen Diagnostik- und Behandlungspfade, eine strukturierte und gezielte Übergabe sowie regelmäßige Fortbildung erforderlich sind.

\section{Schlüsselwörter}

Fokus · Septischer Schock · Infektion · Rettungsdienst · Antiinfektiva

\section{Hinführung zum Thema}

Die Sepsis ist mit einer Inzidenz vonjährlich rund 50 Mio. Neuerkrankungen weltweit eine der Haupttodesursachen. Damit wird gemäß der "Global-burden-of-diseases"Studie jeder fünfte Todesfall durch eine Sepsis verursacht [35]. Der Verlaufgestaltet sich mitunter fulminant und führt zu einer hohen Sterblichkeit [39]. Sowohl die Inzidenz als auch die Sterblichkeit der Sepsis ist deutlich höher als die anderer Notfälle wie z.B. Myokardinfarkt oder Schlaganfall [41].

Definition. Sepsis ist definiert als akut lebensbedrohliche Organdysfunktion, die verursacht wird durch eine dysregulierte, inadäquate Wirtsantwort auf eine Infektion $[9,43]$. 


\section{Sepsis in der prähospitalen Notfallmedizin}

In der Notfallmedizin stellt die Sepsis eine besondere Herausforderung dar, da sich das Syndrom Sepsis in vielfältiger Ausprägung und zunächst nur wenig spezifisch präsentieren kann und aus diesem Grund häufig nicht oder zu spät erkannt wird [23]. Obgleich die Sepsis bereits seit 2016 zu den Tracer-Diagnosen gehört [16], bleibt anzunehmen, dass viele Patienten, die mit vermeintlich "schlechtem Allgemeinzustand (AZ) " unter dem Stichwort "AZ-Verschlechterung" über den Rettungsdienst in ein Krankenhaus eingeliefert werden, in Wirklichkeit eine beginnende oder manifeste Sepsis aufweisen.

Die vergangenes Jahr publizierte S3Leitlinie zur Prävention, Diagnose, Therapie und Nachsorge der Sepsis legt den Fo-

\section{Abkürzungen}

\begin{tabular}{ll} 
AF & Atemfrequenz \\
$A Z$ & Allgemeinzustand \\
BAS & Blutdruck, Atmung, Sauerstoffsätti- \\
& gung $(90$ - 30 - 90) \\
CIS & "Cellular injury score" \\
EUSEM & European Society of Emergency \\
& Medicine \\
EWRS & "Early warning and response system" \\
EWS & "Early warning score" \\
GCS & Glasgow Coma Scale \\
i.v. & Intravenös \\
i.O. & Intraossär \\
IDSA & Infectious Diseases Society of \\
& America \\
k.A. & Keine Angabe \\
kgKG & Kilogramm Körpergewicht \\
MAP & Mittlerer arterieller Druck \\
MEDS & "Mortality in emergency department \\
& sepsis" \\
MEWS & "Modified early warning score" \\
MRST & "Modified Robson screening tool" \\
NEWS & "National early warning score" \\
PEEP & "Positive end-exspiratory pressure" \\
PRESEP & "Prehospital early sepsis detection" \\
PRESS & "Prehospital recognition of severe \\
& sepsis" \\
qSOFA & "Quick sequential organ failure \\
& assessment" \\
RST & "Robson screening tool“ \\
SARS-CoV-2 "Severe acute respiratory syndrome \\
SIRS & coronavirus 2" \\
& Systemisches inflammatorisches \\
SOFA & Responsesyndrom \\
& "Sequential organ failure assess- \\
SOP & ment" \\
SpO & "Standard operating procedure" \\
SSC & Periphere Sauerstoffsättigung \\
ZNS & "Surviving sepsis campaign“ \\
\hline & Zentrales Nervensystem \\
\hline
\end{tabular}

kus auf das intensivmedizinische Setting und geht nicht explizit auf die Besonderheiten der prähospitalen Versorgung von Patienten mit Verdacht auf eine Sepsis ein [9]. Deshalb möchten die Autoren die Gelegenheit nutzen, um die aktuelle klinische Evidenzlage explizit im Hinblick auf die prähospitale Notfallversorgung herauszuarbeiten und daraus Handlungsoptionen für den Notarzt- und Rettungsdienst abzuleiten.

\section{Einsatztaktik und Zeit- management}

Das klinische „1 h bundle" sollte idealerweise innerhalb der ersten Stunde abgeschlossen sein. Trotz fehlender harter Evidenz für die Festlegung eines Zeitpunkts von 60 min nach dem medizinischen Erstkontakt [17] folgt diese Logik dem Prinzip einer "golden hour", wie es bei vielen anderen notfallmedizinischen Entitäten, wie beispielsweise dem Polytrauma, Schlaganfall oder okklusiven Myokardinfarkt, etabliert und akzeptiert ist [9, 12, 15, 16].

Für die prähospitale Versorgung folgt daraus, dass Patienten mit (Verdacht auf) Sepsis nach höchstens $60 \mathrm{~min}$ in einem geeigneten Krankenhaus aufgenommen werden sollen. Dazu gehören nach Überzeugung der Autoren eine über $24 \mathrm{~h}$ besetzte Notaufnahme mit Verfügbarkeit der entsprechenden Fachgebiete (prinzipiell innere Medizin und Chirurgie sowie ggf. Neurologie, Pädiatrie etc.), eine 24h-Verfügbarkeit von Labor (Point-of-CareTestung, zeitnahe Notfallwerte, Erregerdiagnostik) und Bildgebung (insbesondere Computertomographie) sowie eine freie Intensiv- und ggf. Operationskapazität. Die Diagnostik einschließlich der mikrobiologischen Probenentnahme sollte innerhalb von 90 min nach Notrufeingang erfolgen (• Abb. 1, [16]).

\section{Diagnosekriterien}

Sollte sich aus den Angaben des Patienten bzw. der Angehörigen der Verdacht auf das Vorliegen einer Infektion ergeben, sollte dieser durch eine sorgfältige körperliche Untersuchung erhärtet werden. Allerdings ist eine umfassende klinische Untersuchung an der Einsatzstelle häufig bereits aufgrund äußerer Umstände limitiert. Somit kann eine präzise Untersuchung meist erst in der Notaufnahme durchgeführt werden; diese muss dann fundiert erfolgen. Da der Rettungs- und Notarztdienst über die Untersuchung hinaus regelhaft Eindrücke von den (häuslichen) Umgebungsbedingungen erhält, sind Hinweise zum vermuteten Fokus für die Notaufnahme durchaus hilfreich und willkommen - diese dürfen aber nicht zu einem Fixierungsfehler führen.

Daneben treten bei der Sepsis häufig unspezifische Symptome des Gesamtorganismus, wie Fieber, Schüttelfrost, Hyperventilation, Vigilanzminderung, und unspezifische Schockzeichen, wie Tachykardie und Hypotension, oder allgemeines Krankheitsgefühl bis hin zu einem schwerstkranken Gesamteindruck („Facies hippocratica“) auf.

Abhängig vom jeweiligen Fokus sind weitere charakteristische Symptome möglich, als Merkhilfe eignet sich das Akronym "LUCCAASS" (• Tab. 1, [24]).

Früher wurde zur Diagnosestellung einer Sepsis neben den Hinweisen auf eine Infektion das Konzept des "systemischen inflammatorischen Responsesyndroms $(\mathrm{SIRS})^{\prime \prime}[8,22]$ mit den Kriterien

- Hypo-/Hyperthermie,

- Tachykardie,

- Hyperventilation oder Horovitz-Quotient $<200$,

- Leukozytose/-penie oder $>10 \%$ unreife Leukozyten

herangezogen. Auch wenn anhand der SIRS-Kriterien 12,1\% aller Patienten mit Sepsis nicht als solche erfasst werden [19], sollte die Symptomkonstellation im Hinterkopf behalten werden.

In der aktuellen Sepsis-3-Definition [43] wurden die SIRS-Kriterien durch den "Sequential-organ-failure-assessment (SOFA)"-Score (- Tab. 2, [48]) abgelöst. Sollte dieser Score um $\geq 2$ Punkte ansteigen, kann die Diagnose "Sepsis" gestellt werden. Andernfalls sollten zunächst Differenzialdiagnosen abgeklärt und bei fortbestehendem Verdacht eine mögliche Sepsis reevaluiert werden [43].

Treffen bei der Sepsis trotz adäquater Volumensubstitution die beiden folgenden Bedingungen zu, spricht man von einem "septischen Schock" [43]: 


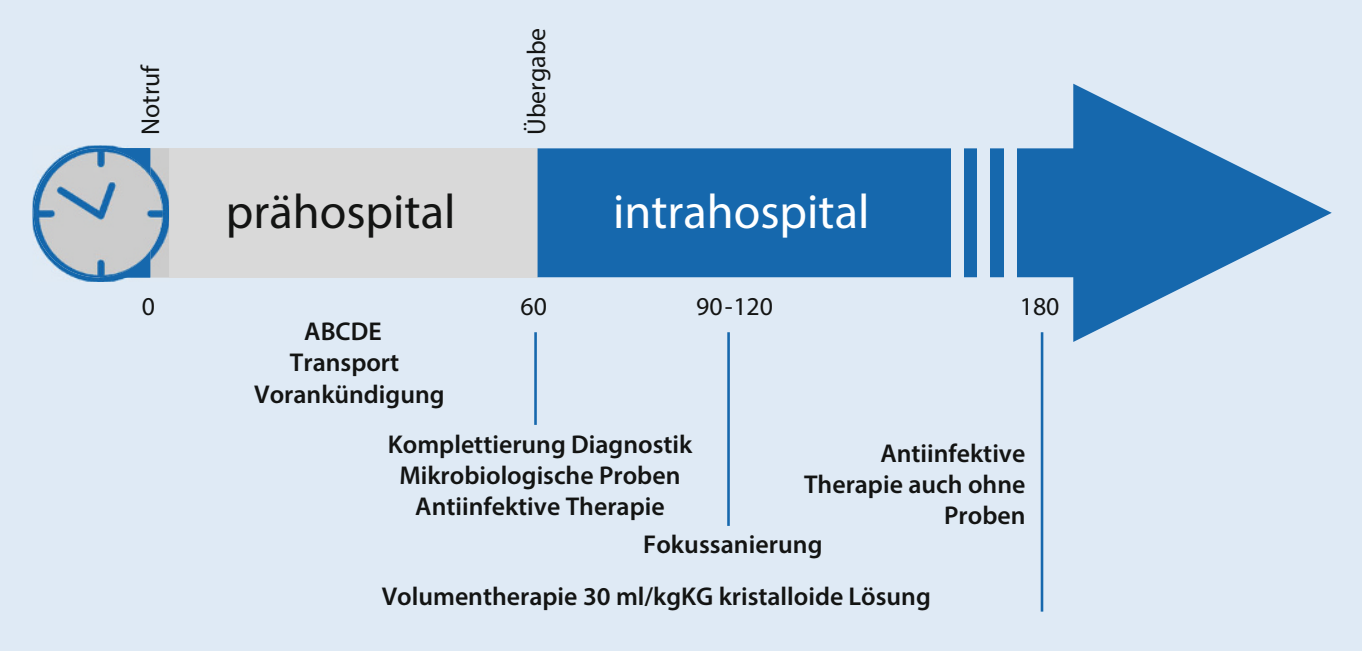

Abb. $1 \triangleleft$ Zeitmanagement in der prä- und intrahospitalen Versorgungsphase bei Sepsisverdacht

1. Vasopressoren sind erforderlich, um einen mittleren arteriellen Druck (MAP) von $\geq 65 \mathrm{~mm} \mathrm{Hg}$ zu erreichen (beeinträchtigte Makrozirkulation);

2. Serumlaktat $\geq 2 \mathrm{mmol} / \mathrm{l}$ ( $\geq 18 \mathrm{mg} / \mathrm{dl}$; beeinträchtigte Mikrozirkulation).

Da die Kalkulation des SOFA-Scores laborchemische Befunde voraussetzt, ist dieser Score insbesondere der innerklinischen intensivmedizinischen Umgebung vorbehalten und damit die definitive Diagnosestellung einer Sepsis erst in der Klinik möglich. Um Patienten mit einem hohen Risiko für das Vorliegen einer Sepsis schneller identifizieren zu können, wurde der "Quick-SOFA(qSOFA)"-Score eingeführt. Dieser eignet sich aufgrund seiner niedrigen Sensitivität allerdings weniger zum Sepsisscreening als vielmehr zur prädiktiven Risikostratifizierung kritisch erkrankter Patienten [1, 9, 30, 43, 46]. Dennoch sollte differenzialdiagnostisch an eine Sepsis gedacht werden, wenn bei einem Infektionsverdacht mindestens 2 der folgenden Kriterien erfüllt sind:

1. Atemfrequenz $(\mathrm{AF}) \geq 22 / \mathrm{min}$,

2. Vigilanzminderung,

3. Hypotension $\leq 100 \mathrm{~mm} \mathrm{Hg}$ systolisch.

Neben dem qSOFA existiert eine Reihe weiterer Frühwarnscores (- Tab. 3), die allesamt eine hohe Sensitivität zur Identifikation kritischer Patienten, jedoch nur eine geringe Spezifität für die Detektion einer Sepsis gemeinsam haben. Prinzipiell erheben diese Scoringsysteme in unterschiedlichen Kombinationen überwiegend Para- meter, die zum minimalen Notfalldatensatz (MIND) der Deutschen Interdisziplinären Vereinigung für Intensiv- und Notfallmedizin (DIVI) zählen und regelhaft in der prähospitalen Notfallmedizin erfasst werden. Darüber hinaus erfassen manche Scores weitere Patienten-, Anamnese- oder Therapiefaktoren oder aber bestimmte Laborparameter. Insbesondere wenn Blutbild oder klinische Chemie zur Errechnung eines Scores erforderlich sind, limitiert dies die Anwendungsmöglichkeit auf die Notaufnahme. Das Vorhandensein bzw. die Ausprägung der einzelnen Items ergibt innerhalb des jeweiligen Scoringsystems eine bestimmte Punktzahl, die ab einem definierten Wert einen potenziell kritischen Patienten identifiziert. Hierunter ist nach Ansicht der Autoren insbesondere der im Vereinigten Königreich etablierte „national early warning score (NEWS) 2" hervorzuheben, der zur Detektion einer Sepsis im notfallmedizinischen Setting dem qSOFAScore überlegen ist [26]. Der NEWS 2 impliziert ab einem kumulierten Absolutwert von $\geq 5$ Punkten eine besondere Vigilanz und dessen Veränderung eignet sich darüber hinaus zur Verlaufsbeobachtung [34]. Häufig bilden die Scoringsysteme weitere Hinweise für das Vorliegen einer Infektion, wie z. B. Herzfrequenz, Hypo- oder Hyperthermie, $a b$.

Bei positivem Score oder wenn trotz negativem Scoring weiterhin eine Sepsis vermutet wird, soll nach Klinikaufnahme der SOFA-Score erhoben werden [9]. Die aktualisierten "surviving sepsis campaign" (SSC)-Guidelines empfehlen nicht mehr die
Nutzung des qSOFA, sondern Scoringsysteme wie MEWS bzw. NEWS (• Tab. 3 [32]).

Praxistipp. Gerade auch prähospital können und sollten bei den verwendeten Scores (mit Ausnahme der Laborbefunde) alle Parameter erhoben und dokumentiert werden.

\section{Therapieprinzipien}

Aufgrund des mitunter fulminanten Verlaufs und der vitalen Bedrohung einerseits und einer langen Dauer bis zum Vorliegen der mikrobiologischen Befunde andererseits müssen bei einer Sepsis diagnostische und therapeutische Schritte unverzüglich und parallel erfolgen. Diese folgen den Empfehlungen der S3-Leitlinie zur Sepsis [9], die sich wiederum an den SSC-Guidelines aus dem Jahr 2017 orientiert, welche kürzlich aktualisiert wurden [32] und und das Vorgehen in „Bündeln“ („,bundles“) zusammenfassen.

Merke. In akut vital bedrohlichen Situationen hat sich das ursprünglich der Traumaversorgung entstammende „ABCDE-Schema" bewährt.

Da sich diese Therapieprinzipien in vielen kritischen Situationen der Notfallmedizin fest etabliert haben und jedem notfallmedizinisch Tätigen geläufig sein sollten, verweisen wir an dieser Stelle auf das Vorgehen und die Priorisierung gemäß $A B C$ $D E$-Schema. Spezifische Punkte innerhalb dieses Schemas, die bei der Versorgung von Patienten mit (Verdacht auf) Sepsis 
Tab. 1 Typische Infektfokusse bei der Sepsis nach "LUCCAASS"-Akronym

\begin{tabular}{|c|c|c|}
\hline Fokus & Symptome & $\begin{array}{l}\text { Häufigkeit }^{\mathrm{a}} \\
\text { in \% }\end{array}$ \\
\hline "Lung" (Lunge) & $\begin{array}{l}\text { Dyspnoe, trockene Rasselgeräusche, Husten, Spu- } \\
\text { tum, Zyanose, atemabhängiger Brustschmerz }\end{array}$ & $51-63$ \\
\hline $\begin{array}{l}\text { "Urine“" (Urogenitalsystem, } \\
\text { Niere) }\end{array}$ & $\begin{array}{l}\text { An-/Oligurie, Schmerzen in Blase oder Flanke, } \\
\text { Klopfschmerz Nierenlager, trüber oder übelrie- } \\
\text { chender Urin }\end{array}$ & $2-18$ \\
\hline $\begin{array}{l}\text { "Central nervous system" } \\
\text { (zentrales Nervensystem) }\end{array}$ & $\begin{array}{l}\text { Kopfschmerz, Vigilanzstörung, Fieber, Meningis- } \\
\text { mus, Lichtempfindlichkeit, Übelkeit, Erbrechen, } \\
\text { Krampfanfall }\end{array}$ & $1-3$ \\
\hline „Cardiac" (Herz) & $\begin{array}{l}\text { Neues Herzgeräusch, Fieber, Rhythmusstörungen, } \\
\text { Hinweise aus Anamnese und Untersuchung auf } \\
\text { kardiale Risikofaktoren, degenerative Herzklappen, } \\
\text { Operation, i.v.-Drogenabusus, einliegende Katheter, } \\
\text { Dialyse, rheumatisches Fieber }\end{array}$ & $1-2$ \\
\hline $\begin{array}{l}\text { „Abdomen“ (Gastroin- } \\
\text { testinaltrakt, Abdomen) }\end{array}$ & $\begin{array}{l}\text { Bauchschmerz, Abwehrspannung, Blumberg-Zei- } \\
\text { chen, hochgestellte oder fehlende Darmgeräusche, } \\
\text { Obstipation, Übelkeit, Erbrechen, Diarrhö, Hinweise } \\
\text { aus Anamnese bzw. Untersuchung auf Operation, } \\
\text { Immunsuppression }\end{array}$ & $10-41$ \\
\hline $\begin{array}{l}\text { "Arthritis" (Gelenke, Im- } \\
\text { plantate) }\end{array}$ & $\begin{array}{l}\text { Schmerz, Funktionseinschränkung, Hinweise aus } \\
\text { Anamnese bzw. Untersuchung auf Operation (z. B. } \\
\text { Narben, Prothesenausweise), Immunsuppression, } \\
\text { Rheumatoide Arthritis }\end{array}$ & $1-2$ \\
\hline $\begin{array}{l}\text { „Skin“ (Binde- und Stütz- } \\
\text { gewebe, Haut) }\end{array}$ & $\begin{array}{l}\text { Sichtbare Wunde, Rötung, Schwellung, Schmerz, } \\
\text { Überwärmung, Funktionseinschränkung }\end{array}$ & $7-10$ \\
\hline „Spine“ (Wirbelsäule) & $\begin{array}{l}\text { Myalgien, Rückenschmerzen, periphere neurologi- } \\
\text { sche Defizite (Parästhesien, Paresen) }\end{array}$ & k. A. \\
\hline (Primäre Bakteriämie) & Unspezifisch & $2-7$ \\
\hline \multicolumn{3}{|c|}{$\begin{array}{l}\text { k. A. keine Angabe } \\
\text { 'Die Häufigkeitsangaben nach [13, 14, 20,39] beziehen sich auf Intensivstationen in Deutschland. Da } \\
\text { nicht jeder Patient mit Sepsis aus der Notaufnahme auf eine Intensivstation verlegt wird, lassen sich } \\
\text { die Häufigkeiten nicht uneingeschränkt auf das prähospitale Setting [5] übertragen }\end{array}$} \\
\hline
\end{tabular}

über die allgemeinen Basismaßnahmen hinausgehen, werden in $\mathbf{A b b} .2$ hervorgehoben.

\section{Anamnese}

Insbesondere bei erforderlicher Analgosedierung oder ggf. Narkoseeinleitung und Intubation gehören die Einsatzkräfte zu den letzten Personen, die den Patienten möglicherweise noch in einem ansprechbaren Zustand antreffen. Deshalb sollte, in zeitlich vertretbarem Umfang, die Anamnese anhand eines Schemas erhoben und dokumentiert werden.

Anamnestisch sollte unter anderem jegliche Form von Immunsuppression (als Folge von Vorerkrankungen, Alter sowie iatrogen oder hereditär) erfragt werden, stellt sie doch einen besonderen Risikofaktor für die Entwicklung einer Infektion und Sepsis dar.

\section{$A$ und $B$ : Atemwege und (Be-)Atmung}

Insbesondere bei pulmonalem Fokus kann eine Sauerstoffgabe, bei zunehmender respiratorischer Erschöpfung eine nichtinvasive Ventilation oder gar die endotracheale Intubation und kontrollierte Beatmung erforderlich werden. Das Vorgehen folgt der Handlungsempfehlung zur prähospitalen Notfallnarkose [6] und der S1-Leitlinie zum prähospitalen Atemwegsmanagement [45].

Praxistipp. Bei ausgeprägter Schocksymptomatik mit gestörter Mikrozirkulation misst die periphere Sauerstoffsättigung $\left(\mathrm{S}_{\mathrm{p}} \mathrm{O}_{2}\right)$ unter Umständen keine validen Werte.

Die Indikation für eine invasive Beatmung sollte kritisch geprüft werden, um die "on-scene time" nicht zu verlängern und die klinische Behandlung der Infekti- on nicht zu verzögern. Andererseits darf dies jedoch nicht dazu führen, dass bei bestehender Indikation eine Intubation unterbleibt und ein Patient die Notaufnahme hypoxisch erreicht. Klare Indikationen zur prähospitalen Notfallnarkose und Atemwegssicherung, die auch für die Sepsis gelten, sind [6]:

- akute respiratorische Insuffizienz

(Hypoxie oder Atemfrequenz < 6 oder $>29 /$ min) bei Kontraindikationen zur bzw. Versagen der nichtinvasiven Ventilation;

- Bewusstlosigkeit oder neurologisches Defizit mit Aspirationsgefahr.

Der Vollständigkeit halber sei an dieser Stelle erwähnt, dass die Handlungsempfehlung zur prähospitalen Notfallnarkose zudem das schwere bzw. Polytrauma mit hämodynamischer Instabilität (Blutdruck $<90 \mathrm{~mm} \mathrm{Hg}$ systolisch), Hypoxie $\left(\mathrm{S}_{\mathrm{p}} \mathrm{O}_{2}\right.$ $<90 \%$ trotz $\mathrm{O}_{2}$-Gabe) oder Schädel-HirnTrauma (GCS < 9) als Intubationsindikation nennt [6].

Wird zur Sicherung der Atemwege und Beatmung eine Intubation und damit eine Narkoseeinleitung erforderlich, sind das Risiko einer Hypotension und $\mathrm{Hy}$ poxie sowie die prähospital erschwerten Umgebungsbedingungen zu bedenken. Die Atemwegssicherung muss zeitkritisch und zwingend erfolgreich durchgeführt werden [45].

Praxistipp. Propofol sollte als Induktionshypnotikum aufgrund seiner kreislaufdepressiven Wirkung bei hämodynamisch instabilen Patienten unbedingt vermieden werden. Auch Etomidat ist aufgrund der Suppression von Kortisol- und Aldosteronsynthese in diesen Fällen nicht geeignet. Die Autoren empfehlen die Narkoseinduktion, so sie denn erforderlich werden sollte, mit Esketamin, Midazolam, gegebenenfalls ergänzt um ein Opioid, sowie einem schnell anschlagenden Muskelrelaxans.

Die kontrollierte Beatmung folgt dem Konzept der „lungenprotektiven Beatmung" mit Tidalvolumina von $6-8 \mathrm{ml}$ kgKG bei endinspiratorischem Atemwegsdruck von $\leq 30 \quad \mathrm{~cm} \mathrm{H}_{2} \mathrm{O}$ bzw. "driving pressure" $\leq 15 \mathrm{~cm} \mathrm{H}_{2} \mathrm{O}$ sowie einem PEEP von $\geq 5 \mathrm{~cm} \mathrm{H}_{2} \mathrm{O}$ [9]. 


\begin{tabular}{|c|c|c|c|c|c|}
\hline Organsystem & o Punkte & 1 Punkt & 2 Punkte & 3 Punkte & 4 Punkte \\
\hline \multicolumn{6}{|l|}{ Atmungssystem } \\
\hline Horovitz-Quotient & $\begin{array}{l}\geq 400 \mathrm{~mm} \mathrm{Hg} \\
(\geq 53,3 \mathrm{kPa})\end{array}$ & $\begin{array}{l}<400 \mathrm{~mm} \mathrm{Hg} \\
(<53,3 \mathrm{kPa})\end{array}$ & $\begin{array}{l}<300 \mathrm{~mm} \mathrm{Hg} \\
(<40 \mathrm{kPa})\end{array}$ & $\begin{array}{l}<200 \mathrm{~mm} \mathrm{Hg} \\
(<26,7 \mathrm{kPa})\end{array}$ & $\begin{array}{l}<100 \mathrm{~mm} \mathrm{Hg} \\
(<13,3 \mathrm{kPa})\end{array}$ \\
\hline \multicolumn{6}{|l|}{ Gerinnung } \\
\hline Thrombozyten & $\geq 150 / \mathrm{nl}$ & $<150 / \mathrm{nl}$ & $<100 / \mathrm{nl}$ & $<50 / \mathrm{nl}$ & $<20 / \mathrm{nl}$ \\
\hline \multicolumn{6}{|l|}{ Leber } \\
\hline Bilirubin & $\begin{array}{l}<1,2 \mathrm{mg} / \mathrm{dl} \\
(<20 \mu \mathrm{mol} / \mathrm{l})\end{array}$ & $\begin{array}{l}1,2-1,9 \mathrm{mg} / \mathrm{dl} \\
(20-32 \mu \mathrm{mol} / \mathrm{l})\end{array}$ & $\begin{array}{l}2,0-5,9 \mathrm{mg} / \mathrm{dl} \\
(33-101 \mu \mathrm{mol} / \mathrm{l})\end{array}$ & $\begin{array}{l}6,0-11,9 \mathrm{mg} / \mathrm{dl} \\
(102-204 \mu \mathrm{mol} / \mathrm{l})\end{array}$ & $\begin{array}{l}\geq 12,0 \mathrm{mg} / \mathrm{dl} \\
(\geq 204 \mu \mathrm{mol} / \mathrm{l})\end{array}$ \\
\hline \multicolumn{6}{|c|}{ Kardiovaskuläres System } \\
\hline $\begin{array}{l}\text { Katecholamine (Lauf- } \\
\text { rate über } \geq 1 \mathrm{~h} \text { ) }\end{array}$ & $\begin{array}{l}\text { MAP } \\
\geq 70 \mathrm{~mm} \mathrm{Hg}\end{array}$ & $\mathrm{MAP}<70 \mathrm{~mm} \mathrm{Hg}$ & $\begin{array}{l}\text { Dopamin } \leq 5 \mu \mathrm{g} / \mathrm{kgKG} \\
\text { und Minute oder } \\
\text { Dobutamin (dosisunab- } \\
\text { hängig) }\end{array}$ & $\begin{array}{l}\text { Dopamin } 5,1-15 \mu \mathrm{g} / \mathrm{kgKG} \\
\text { und Minute oder } \\
\text { Adrenalin } \leq 0,1 \mu \mathrm{g} / \mathrm{kgKG} \\
\text { und Minute oder Nor- } \\
\text { adrenalin } \leq 0,1 \mu \mathrm{g} / \mathrm{kgKG} \\
\text { und Minute }\end{array}$ & $\begin{array}{l}\text { Dopamin }>15 \mu \mathrm{g} / \mathrm{kgKG} \\
\text { und Minute oder Adrena- } \\
\text { lin }>0,1 \mu \mathrm{g} / \mathrm{kgKG} \text { und } \\
\text { Minute oder Noradre- } \\
\text { nalin }>0,1 \mu \mathrm{g} / \mathrm{kgKG} \text { und } \\
\text { Minute }\end{array}$ \\
\hline \multicolumn{6}{|l|}{ Zentrales Nervensystem } \\
\hline GCS & 15 & $13-14$ & $10-12$ & $6-9$ & $<6$ \\
\hline \multicolumn{6}{|l|}{ Niere } \\
\hline Kreatinin & $\begin{array}{l}<1,2 \mathrm{mg} / \mathrm{dl} \\
(<110 \mu \mathrm{mol} / \mathrm{l})\end{array}$ & $\begin{array}{l}1,2-1,9 \mathrm{mg} / \mathrm{dl} \\
(110-170 \mu \mathrm{mol} / \mathrm{l})\end{array}$ & $\begin{array}{l}2,0-3,4 \mathrm{mg} / \mathrm{dl} \\
(171-299 \mu \mathrm{mol} / \mathrm{l})\end{array}$ & $\begin{array}{l}3,5-4,9 \mathrm{mg} / \mathrm{dl} \\
(300-440 \mu \mathrm{mol} / \mathrm{l})\end{array}$ & $\begin{array}{l}\geq 5,0 \mathrm{mg} / \mathrm{dl} \\
(\geq 440 \mu \mathrm{mol} / \mathrm{l})\end{array}$ \\
\hline Diurese & - & - & - & $<500 \mathrm{ml} / \mathrm{d}$ & $<200 \mathrm{ml} / \mathrm{d}$ \\
\hline
\end{tabular}

\section{C: Kreislauf}

Diehämodynamische Stabilisierung nimmt bei Patienten mit (vermuteter) Sepsis bereits im prähospitalen Setting einen zentralen Stellenwert ein [9, 15].

Praxistipp. Solange keine invasive arterielle Blutdruckmessung erfolgen kann, ist unbedingt auf engmaschige (Messintervall $<5 \mathrm{~min}$ ) Kontrollen von Blutdruck und Rekapillarisierungszeit zu achten, um zuverlässig einen MAP von $\geq 65 \mathrm{~mm} \mathrm{Hg}$ bzw. systolischen Blutdruck $\geq 100 \mathrm{~mm} \mathrm{Hg}$ aufrechterhalten zu können. Hierzu sind in der Regel mehrere intravenöse (i.v.-)Zugänge erforderlich. Ist dies durch Schocksymptomatik, Gefäßsituation oder Ödembildung absehbar nicht realisierbar, kann auch ein intraossärer (i.o.-)Zugang geschaffen werden. Hierbei ist allerdings zu beachten, dass der Fluss über einen i.o.-Zugang erfahrungsgemäß meist nicht für eine Volumentherapie im Sinne der im Folgenden vorgestellten "fluid resuscitation“ ausreicht und damit die "C-Problematik" allein nicht löst. In der Klinik wird in der Regel ein zentralvenöser Zugangsweg mit großen Lumina etabliert. Auf die Sinnhaftigkeit einer vorübergehenden Applikation von Vasopressoren über periphere i.v.-Zugänge wird in den neuen SSC-Guidelines explizit hingewiesen, um den Beginn der vasoaktiven Therapie (und die dadurch erhoffte Kreislaufstabilisierung) nicht bis zur Anlage eines zentralen Venenkatheters zu verzögern [15].

Neben Zeichen einer Exsikkose interessiert beim Hautbefund das Vorliegen einer Marmorierung („,mottling ${ }^{\prime \prime}$ ) an Knie- und Ellenbogengelenk, auf die bereits prähospital geachtet werden sollte. Die Marmorierung ist Abbild der Mikrozirkulationsstörung, die Ausprägungsschwere stellt einen negativen Prognoseprädiktor dar [2].

Praxistipp. Mit der zunehmenden Verbreitung portabler Ultraschallgeräte im Notarztdienst kann der kardiozirkulatorische Status auch sonographisch („point of care ultrasound", POCUS) schnell und zuverlässig abgeschätzt und im Verlauf kontrolliert werden.

\section{Laktatmessung}

Die Messung des Laktats im Serum dient als Surrogatparameter der Mikrozirkulation sowie als Ausgangspunkt zur weiteren Verlaufsbeobachtung und Steuerung der hämodynamischen Stabilisierung (,resuscitation") [15]. Die Aufnahme des Laktatwerts in die Definition des septischen Schocks wird häufig als Kritikpunkt angeführt, da die Messung dieses Laborparameters nicht immer und überall verfügbar ist [25]. Die „Point-of-Care“-Messung des Laktats kann mit einfachen Geräten und Teststreifen erfolgen und entspricht im Vorgehen der bereits etablierten Blutzuckermessung. Dies ist auch im Rettungsdienst prinzipiell möglich und aus Sicht der Autoren sinnvoll. Mit der Verfügbarkeit portabler Blutgasanalysegeräte können bereits am Einsatzort eine vollständige Blutgasanalyse und über Laktat und Blutzucker hinaus auch Sauerstoff- bzw. Kohlendioxidpartialdrücke sowie der Hämoglobingehalt gemessen und hierdurch differenzialdiagnostisch und therapeutisch bedeutsame Hinweise bei kritischen Patienten geliefert werden.

Die ANDROMEDA-SHOCK-Studie konnte zeigen, dass die Steuerung der Hämodynamik anhand der Rekapillarisierungszeit („'capillary refill time“) verglichen mit der Steuerung nach den Laborwerten des Serumlaktats hinsichtlich der Überlebensraten zumindest gleichwertig und bezogen auf die Häufigkeit von Organversagen 
Tab. 3 Scoringsysteme zur Erfassung einer Sepsis bzw. einer kritischen Erkrankung ${ }^{\mathrm{a}}$

\begin{tabular}{|c|c|c|c|c|c|c|c|c|c|c|c|}
\hline & SIRS & SOFA & qSOFA & {$[\mathrm{M} / \mathrm{N}] \mathrm{EWS}$} & PRESEP & BAS & [M]RST & MEDS & CIS & PRESS & EWRS \\
\hline Patientenfaktoren & - & - & - & - & - & - & - & $x$ & $x$ & $x$ & - \\
\hline Anamnesekriterien & - & - & - & - & - & - & $x$ & $x$ & - & - & - \\
\hline Therapiefaktoren & - & $x$ & - & {$[\mathrm{X}]$} & - & - & - & - & - & - & - \\
\hline \multicolumn{12}{|l|}{ Vitalparameter } \\
\hline Atemfrequenz & $x$ & - & $x$ & $x$ & $x$ & $x$ & $x$ & $x$ & $x$ & - & $x$ \\
\hline Sauerstoffsättigung & - & - & - & $x$ & $x$ & $x$ & {$[\times]$} & - & $x$ & $x$ & - \\
\hline Herzfrequenz & $x$ & - & - & $x$ & $x$ & - & $x$ & $x$ & $x$ & - & $x$ \\
\hline Blutdruck & - & $x$ & $x$ & $x$ & $x$ & $x$ & {$[\times]$} & - & $x$ & $x$ & $x$ \\
\hline Bewusstsein & - & $x$ & $x$ & $x$ & $x$ & - & $x$ & $x$ & $x$ & - & - \\
\hline Körpertemperatur & $x$ & - & - & $x$ & $x$ & - & $x$ & $x$ & - & $x$ & $x$ \\
\hline \multicolumn{12}{|l|}{ Laborparameter } \\
\hline Blutzucker & - & - & - & - & $x$ & - & $x$ & - & - & - & - \\
\hline Blutgasanalyse & $x$ & $x$ & - & - & - & - & {$[\times]$} & - & - & - & $x$ \\
\hline Blutbild & $x$ & $x$ & - & - & - & - & - & $x$ & - & - & $x$ \\
\hline Klinische Chemie & - & $x$ & - & - & - & - & - & - & - & - & - \\
\hline \multicolumn{12}{|c|}{ 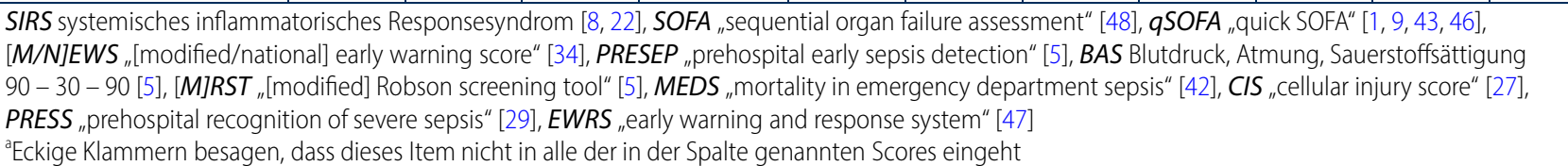 } \\
\hline
\end{tabular}

sogar überlegen ist. Demzufolge könnte im prähospitalen Setting die Volumentherapie anhand des einfach zu erhebenden Markers „Rekapillarisierungszeit" evaluiert werden, wobei das Ziel das Erreichen einer Rekapillarisierungszeit von $<3$ s darstellt. Bei der Interpretation dieser Studienergebnisse ist allerdings zu berücksichtigen, dass diese für die Detektion eines Überlebensvorteils von absolut $15 \%$ bei diesem komplexen Krankheitsbild "underpowered" erscheint [17]. Konsequenterweise empfehlen die aktualisierten SSC-Guidelines explizit die (ergänzende) Nutzung der Rekapillarisierungszeit zur Steuerung der Volumentherapie [32].

Merke. Prähospital kann anstelle des Laktats auch die Rekapillarisierungszeit zur Beurteilung der Mikrozirkulation herangezogen werden.

\section{„Fluid resuscitation"}

Zur Stabilisierung der Hämodynamik wird bei hypotensiven Kreislaufverhältnissen ein Bolus von $30 \mathrm{ml} / \mathrm{kgKG}$ balancierter Vollelektrolytlösung innerhalb der ersten $3 \mathrm{~h}$ appliziert [15, 37].
Praxistipp. Die „fluid resuscitation“ kann bereits prähospital begonnen werden, wenn eine Sepsis die naheliegendste Diagnose ist und kein Anhalt für eine Volumenüberladung besteht.

Die reine „physiologische“ Kochsalzlösung ( $\mathrm{NaCl} 0,9 \%)$ ist obsolet und unter den kolloidalen Infusionslösungen ist Hydroxyethylstärke kontraindiziert. Andere osmolar wirksame Infusionslösungen (Gelatine, Albumin) sind allenfalls in einer späteren Phase sinnvoll, falls sich der Patient mit balancierten kristalloiden Infusionslösungen und Katecholaminen hämodynamisch nicht stabilisieren lässt. Weitere Flüssigkeitsgaben können in Erwägung gezogen werden, wenn weiterhin Hinweise für eine Hypoperfusion vorliegen. Im prähospitalen Setting können hierfür unter anderem Herzfrequenz, Blutdruck, Rekapillarisierungszeit, Sauerstoffsättigung und Atemfrequenz herangezogen werden; ein darüber hinausgehendes erweitertes hämodynamisches Monitoring ist prähospital nicht regelhaft verfügbar.

Bei manifestem Schock ist in der Regel neben der Flüssigkeitstherapie auch der frühzeitige Einsatz von Vasopressoren erforderlich, um einen MAP von $65 \mathrm{~mm} \mathrm{Hg}$ schnellstmöglich zu erreichen und damit die Perfusion aufrechtzuerhalten.
Praxistipp. Das Katecholamin der Wahl ist in diesem Fall Noradrenalin, beginnend mit Boli à $10 \mu \mathrm{g}$; im Verlauf scheint auch prähospital die Etablierung einer Spritzenpumpe sinnvoll.

Sollte hierdurch keine ausreichende hämodynamische Stabilität erreicht werden, können zusätzlich Adrenalin oder das prähospital meist nicht verfügbare Vasopressin sowie in einem weiteren Eskalationsschritt Dobutamin eingesetzt werden. Bei letzterem ist eine periphere Vasodilatation zu bedenken, die ausgeprägte Erhöhung des Herzzeitvolumens bei septischer Kardiomyopathie ist hauptsächlich auf eine Zunahme der Herzfrequenz zurückzuführen [9, 15, 37].

Merke. Zur Kreislaufstabilisierung werden balancierte kristalloide Infusionslösungen verwendet und zur Aufrechterhaltung der Perfusion ggf. frühzeitig um Katecholamine (Noradrenalin) ergänzt.

\section{D: neurologischer Status}

Der neurologische Status einschließlich Blutglukose ist prähospital auch bei der Sepsis unbedingt zu erheben und sorgfältig zu dokumentieren, sodass Veränderungen im Verlauf bemerkt werden 


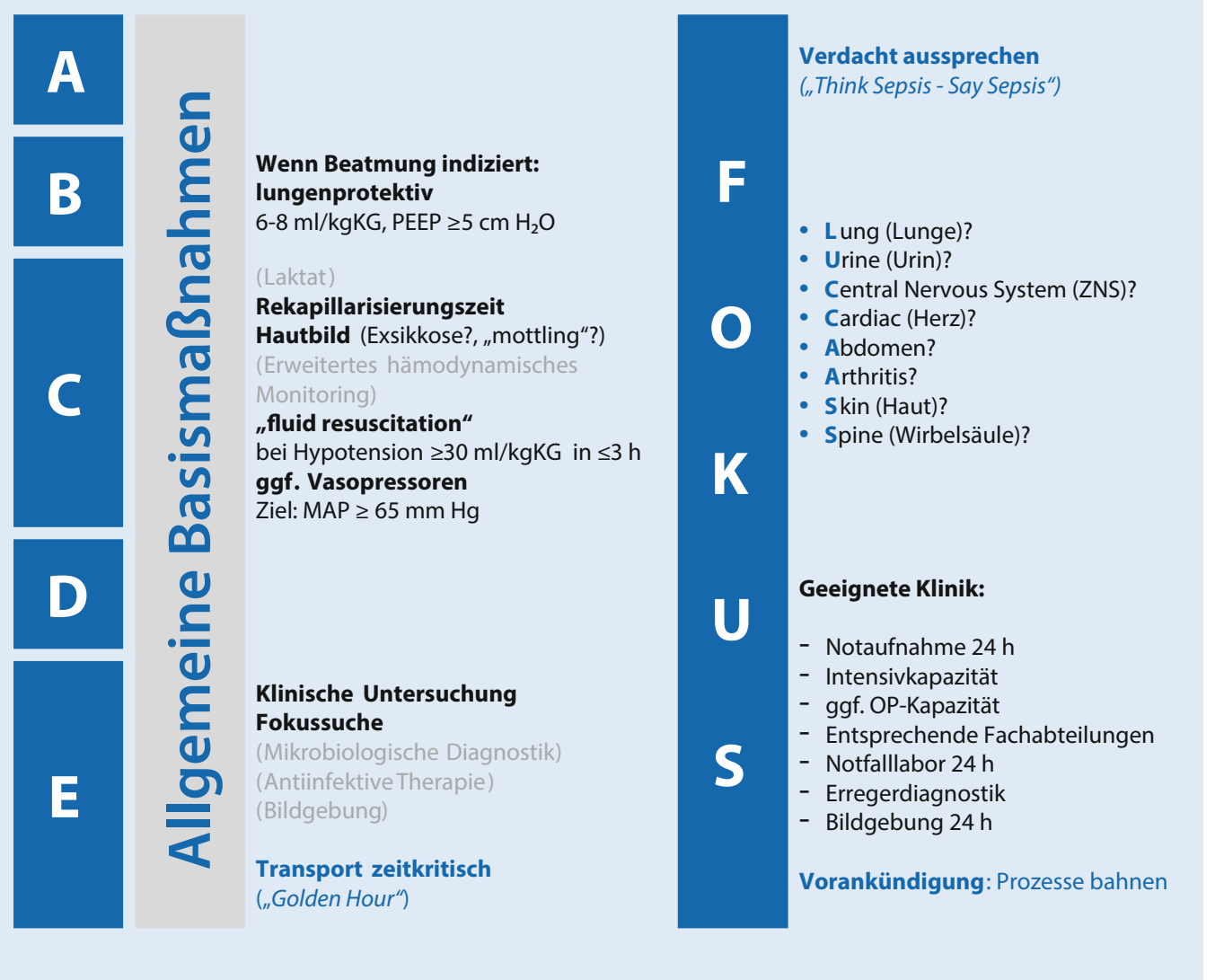

Abb. $2 \triangleleft$ Maßnahmenbündel nach „ABCDE-Schema" mit Hervorhebung der prähospitalen sepsisspezifischen Therapie. In Klammern und hellgrauer Schrift sind Maßnahmen erwähnt, die prähospital nicht regelhaft verfügbar sind. $P E E P$ „positive end-exspiratory pressure", $M A P$ mittlerer arterieller Druck, ZNS zentrales Nervensystem können. Parameter wie GCS und Blutglukosekonzentration gehen zudem in unterschiedliche diagnostische Scores ein (- Tab. 2 und 3).

\section{E: Exploration und Umgebungs- bedingungen}

Die während der prähospitalen Versorgungsphase erhobenen Untersuchungsbefunde einschließlich Körpertemperatur und Hautbefund (z.B. Exsikkose, "mottling" - s.oben) können ebenso wie die beobachteten Umgebungsbedingungen wertvolle Hinweise für das weitere Vorgehen liefern.

\section{Mikrobiologische Diagnostik}

Zur mikrobiologischen Diagnostik gehört die Abnahme von Blutkulturen und je nach vermutetem Fokus ggf. die erweiterte Erregerdiagnostik, beispielsweise im Urin, respiratorischen Sekret, Liquor oder in Abstrichen. Dies soll grundsätzlich vor Beginn einer antimikrobiellen Therapie erfolgen, diese jedoch auch nicht verzö- gern $[9,15]$. Die Gewinnung mikrobiologischer Proben ist im Rettungsdienst prinzipiell möglich [49], setzt jedoch streng sterile Bedingungen voraus. Als weiteres Problem werden beispielsweise Blutkulturen nur in 2,6\% der Rettungsdienstbereiche überhaupt vorgehalten [11] und die prähospital genutzten Produkte könnten möglicherweise nicht mit denen der Klinik übereinstimmen und damit das weitere prozedurale Verfahren beeinflussen. Dies schränkt die Fokussuche (-Tab. 1) häufig auf eine sorgfältige Anamnese und körperliche Untersuchung ein und damit auch die Wahrscheinlichkeit, eine Sepsis an der Einsatzstelle als solche zu erkennen. Die Limitationen im prähospitalen Bereich erklären jedoch nicht, weshalb bei jedem 5. Patienten mit Sepsis in Deutschland überhaupt keine Blutkultur abgenommen wird. Nur etwa ein Drittel aller bei Sepsis entnommenen Blutkulturen liefert tatsächlich auch einen mikrobiologischen Befund (- Tab. 4, [39]).

Außerdem ist zu berücksichtigen, dass nicht nur Bakterien, sondern z. B. auch Pilze und Viren (als aktuelles Beispiel das Coro- navirus SARS-CoV-2) eine Sepsis verursachen können. Je nach vermutetem Erreger ist ggf. eine Spezialdiagnostik erforderlich.

\section{Antiinfektive Therapie}

Die Auswahl der kalkulierten antiinfektiven Therapie bei unklarem Fokus richtet sich nach klinischer Symptomatik, antimikrobieller Vorbehandlung sowie Resistenzlage und soll so schnell wie möglich erfolgen [7, 33]. Jede Stunde, die der Beginn der Antibiose verzögert wird, erhöht die Mortalität der Sepsis [21, 40]. Ob der Beginn einer antiinfektiven Therapie bereits am Einsatzort im Hinblick auf das Behandlungsergebnis der Patienten sinnvoll ist, wird kontrovers diskutiert. So konnten einige Pilot- bzw. Machbarkeitsstudien zeigen, dass die Abnahme von Blutkulturen und die Antibiotikagabe sowohl in auf "paramedics" basierten [49] als auch in notarztbasierten Systemen praktikabel sind und einen Zeitvorteil bieten können $[12,38]$. So werden in $26 \%$ der deutschen Rettungsdienstbereiche Antibiotika vorgehalten; überwiegend Cephalosporine und zu einem deutlich ge- 
Tab. 4 Typische Erreger bei der Sepsis

\begin{tabular}{|c|c|}
\hline Erreger & $\begin{array}{l}\text { Häufig- } \\
\text { keit }^{a} \\
\text { in } \%\end{array}$ \\
\hline $\begin{array}{l}\text { Gramnegative Bakterien } \\
\text { - Escherichia coli } \\
\text { - Bacteroides species } \\
\text { - Pseudomonas aeruginosa } \\
\text { - Klebsiella pneumoniae } \\
\text { - Enterobacter species }\end{array}$ & $33-73$ \\
\hline $\begin{array}{l}\text { Grampositive Bakterien } \\
\text { - Enterococcus species } \\
\text { - Koagulasenegative Staphylo- } \\
\quad \text { kokken } \\
\text { - Staphylococcus aureus } \\
\text { - Streptococcus viridans } \\
\text { - Clostridium difficile }\end{array}$ & $23-56$ \\
\hline Pilze, Hefen & \multirow[t]{3}{*}{$4-26$} \\
\hline - Candida species & \\
\hline - Aspergillus fumigatus & \\
\hline Anaerobier & \multirow[t]{3}{*}{$3-4$} \\
\hline - Clostridium difficile & \\
\hline - Bacteroides fragilis & \\
\hline Viren & \multirow[t]{7}{*}{$2-3$} \\
\hline - Cytomegalovirus & \\
\hline - Influenza & \\
\hline - Herpes-simplex-Virus & \\
\hline - Adenovirus & \\
\hline $\begin{array}{l}\text { - Respiratorisches Synzytial- } \\
\text { Virus }\end{array}$ & \\
\hline - Coronavirus & \\
\hline Legionellen & \multirow[t]{2}{*}{$<1$} \\
\hline - Legionella pneumophila & \\
\hline Mykobakterien & \multirow[t]{2}{*}{$<1$} \\
\hline - Mycobacterium tuberculosis & \\
\hline $\begin{array}{l}\text { 'Die Häufigkeitsangaben nach [13, } \\
\text { 39] beziehen sich auf Intensivstatio } \\
\text { Deutschland. Da nicht jeder Patien } \\
\text { aus der Notaufnahme auf eine Inte } \\
\text { verlegt wird, lassen sich die Häufig } \\
\text { uneingeschränkt auf das prähospit } \\
\text { übertragen }\end{array}$ & $\begin{array}{l}4,20, \\
\text { en in } \\
\text { mit Sepsis } \\
\text { sivstation } \\
\text { eiten nicht } \\
\text { e Setting }\end{array}$ \\
\hline
\end{tabular}

ringeren Anteil Penicilline oder sonstige Wirkstoffgruppen [11].

Ob der prähospitale Beginn einer antiinfektiven Therapie auch mit höheren Überlebensraten einhergeht, lässt sich derzeit noch nicht abschließend beantworten. Die „PHANTASi-Studie“ war die erste multizentrische randomisierte kontrollierte Studie, die den Nutzen einer bereits durch Rettungsfachpersonal begonnenen Antibiose bei 2698 Patienten mit vermuteter Sepsis in den Niederlanden untersuchte. Die prähospitale Applikation eines Cephalosporins der Gruppe 3 ging zwar mit ei- nem Zeitvorteil von 26 min vor Eintreffen in der Notaufnahme (zuzüglich im Median 70 min bis zum Beginn der Antibiotikagabe), nicht aber mit einer geringeren Sterblichkeit einher. Diese betrug sowohl in der Interventions- als auch in der Kontrollgruppe $8 \%$ nach 28 Tagen, was für die Sepsis eine extrem niedrige Mortalität darstellt und dadurch möglicherweise die Effektivität einer prähospitalen Antibiotikagabe verschleiern könnte [3]. Überlegungen, inwiefern dieses Ergebnis auch mit der Auswahl des Antibiotikums zusammenhängt und ob andere Wirkstoffgruppen (z.B. Acylaminopenicilline mit $\beta$-Laktamase-Inhibitor oder Carbapenem) möglicherweise zu anderen Ergebnissen geführt hätten, bleiben rein spekulativ. Bei prähospitaler Gabe eines Antibiotikums kann die mikrobiologische Diagnostik am Einsatzort, wie im vorigen Abschnitt ausgeführt, mit Limitationen verbunden oder erst in der Klinik vollständig möglich sein. Eine Abnahme von Blutkulturen nach Beginn der Antibiose reduziert jedoch die ohnehin geringe Positivitätsrate nochmals massiv auf rund $27 \%$ [36].

Auch in der aktuellen Version empfehlen die SSC-Guidelines den sofortigen Beginn der Antibiose, idealerweise innerhalb von einer Stunde nach Erkennen des Krankheitsbildes. Die Evidenz für diese Empfehlung ist jedoch niedrig (Septischer Schock) bzw. sehr niedrig (Sepsis ohne Schock) und die Daten stammen überwiegend aus amerikanischen Notaufnahmen, so dass diese nicht uneingeschränkt auf den prähospitalen Bereich übertragbar sind [15].

Eine Stellungnahme der European Society of Emergency Medicine (EUSEM) kritisiert die Fixierung auf ein starres „1 $\mathrm{h}$ bundle": So sei zwar die frühe Antibiotikagabe eine der wenigen Maßnahmen innerhalb der Sepsisbündel, die mit der Mortalität korrelieren, doch solle gleichzeitig eine unnötige Antibiose (und Volumentherapie) vermieden werden. Ohne den Faktor Zeit in Abrede zu stellen, empfiehlt die EuSEM, mit der Antibiose erst dann zu beginnen, wenn die Diagnose "Sepsis" die wahrscheinlichste ist, und das Ziel von einer Stunde ab Triage für sorgfältig selektierte Patienten anzustreben [17]. In einer aktuellen Empfehlung spricht sich auch die Infectious Diseases Society of America
(IDSA) dementsprechend dafür aus, lediglich beim septischen Schock, nicht aber bei der Sepsis bereits innerhalb des "1 $\mathrm{h}$ bundle" mit der antiinfektiven Therapie zu beginnen [32]. Insgesamt bleibt festzuhalten, dass die Evidenzlage für den Nutzen einer prähospitalen Antibiotikagabe aufgrund der Limitationen an der Einsatzstelle einerseits und nicht ausreichend aussagekräftiger Studien andererseits für eine abschließende Bewertung derzeit noch unzureichend ist. Weitere Studien werden die Frage beantworten müssen, ob der prähospitale Beginn einer antiinfektiven Therapie auch mit einem verbesserten Behandlungsergebnis einhergeht.

In Anlehnung an die „door-to-needle time" beim ischämischen Schlaganfall bzw. "door-to-balloon time" beim okklusiven Myokardinfarkt sollte bei der Sepsis durch die Schaffung entsprechender prä- und intrahospitaler Strukturen (beispielsweise ein "Nichttraumaschockraum" mit angemessenen personellen Ressourcen, konkreten und praktikablen Standardarbeitsanweisungen [,standard operating procedure", SOP] sowie regelmäßigem theoretischem und praktischem Training) nach präziser Voranmeldung eine möglichst kurze "door-to-antibiotic time" erreicht werden $[15,28]$.

Merke. Ein prähospitaler Beginn der antiinfektiven Therapie wird aufgrund der gegenwärtig limitierten Evidenzlage kontrovers diskutiert.

\section{Schnittstelle Notaufnahme}

Eine effiziente Kommunikation, insbesondere an kritischen Schnittstellen wie der Notaufnahme, mit einer sorgfältigen und vollständigen Übergabe ist entscheidend, um die weiteren Behandlungspfade zu triggern. Hierdurch wird die adäquate Weiterversorgung mit gezielter Therapie und schnellstmöglicher Fokussanierung ermöglicht [15].

Um die Sepsis als solche erkennen zu können, müssen die für die Diagnosekriterien relevanten Befunde auch erhoben werden. Bedauerlicherweise werden diese wichtigen Parameter, die direkte therapeutische Konsequenzen haben und das weitere innerklinische Management be- 
einflussen können, zu einem erschreckend hohen Anteil nicht erhoben [10].

\section{Dokumentation}

Das weiterversorgende Team der Notaufnahme bzw. Intensivstation steht im Verlauf häufig vor der Herausforderung, Patienten zu versorgen, über die nur wenig bekannt ist. Insbesondere wenn eine Analgosedierung oder ggf. Narkose und Beatmung erforderlich geworden sind, ist die Beschaffung von Informationen über den Patienten häufig mit einem hohen Aufwand verbunden. Deshalb kommt einer präzisen, sorgfältigen und umfassenden Dokumentation durch das prähospital versorgende Team ein besonderer Stellenwert zu.

Praxistipp. Für die weiterbehandelnden Kollegen können neben der strukturierten Anamnese auch der Allgemeinzustand des Patienten vor dem Notfallereignis, die Kontaktdaten eines Angehörigen und ggf. eine vorliegende Vorsorgevollmacht und/ oder Patientenverfügung hilfreich sein.

\section{Besonderheiten der intra- hospitalen Notfallversorgung}

Unmittelbar nach Klinikaufnahme erfolgt die Komplettierung der klinischen und mikrobiologischen Diagnostik. Je nach Fokus und erwartetem Erregerspektrum wird spätestens jetzt die kalkulierte antiinfektive Therapie begonnen [9].

\section{Fokussanierung}

Das klinische „1 $\mathrm{h}$ bundle" schließt mit der Evaluation einer möglichen Fokussanierung, die höchste Priorität genießt und sobald als logistisch und medizinisch möglich erfolgen soll $[9,15]$. Anhand der Arbeitshypothese und des antizipierten Fokus sollte eine adäquate Zielklinik mit Bedacht gewählt und vorab informiert werden, um bereits die weiteren Behandlungspfade $z u$ triggern und die Übernahme in einer geeigneten Umgebung (z.B. Aufnahmearbeitsplatz der Intensivstation oder Schockraum) vorbereiten zu können. Die Übergabe durch Notarzt bzw. Rettungsdienst an die weiterbehandelnde Klinik stellt eine kritische Schnittstelle dar, die jedoch für eine unmittelbare, strukturierte und gezielte Weiterversorgung der Patienten essenziell ist.

Merke. Die Fokussanierung genießt höchste Priorität, sodass ein zeitkritischer Transport in eine geeignete Klinik erfolgen soll. Durch eine rechtzeitige Vorabinformation können die weiteren Behandlungspfade getriggert werden.

\section{Strukturelle Vorkehrungen}

\section{Prävention}

Die Einhaltung der Hygienestandards und multimodalen Strategien zur Infektionsprävention, darunter insbesondere die Compliance zur Händehygiene, spielen bei der Prävention der Sepsis im Rettungsdienst eine zentrale Rolle. Durch Impfungen entsprechend der Empfehlungen der Ständigen Impfkommission am Robert Koch-Institut kann die Ausbreitung von Infektionserkrankungen eingedämmt werden und es liegt an den Beschäftigten im Gesundheitssystem, zum Wohl der Patienten und auch zum Eigenschutz hier mit gutem Beispiel voranzugehen [44].

\section{Organisation und Ausbildung}

Alle Einsatzkräfte sollten in Bezugauf Infektionsprävention und Früherkennung der Sepsis, wie dies bei anderen Entitäten wie der Reanimation oder dem Polytrauma bereits fest etabliert ist, regelmäßig theoretisch und praktisch fortgebildet werden [9, 15]. Das konkrete Vorgehen sollte strukturierten Algorithmen im Sinne von SOP folgen, die auf die prä- und intrahospitalen Ressourcen vor Ort abgestimmt sind. Obwohl hierfür allgemeine Handlungsanweisungen der Berufsverbände bzw. Behörden vorliegen [4, 31], nutzen nur $10 \%$ der Rettungsdienstbereiche Algorithmen für die Therapie der Sepsis [11]. Im Rahmen des Qualitätsmanagements sollte die Versorgung von Patienten mit vermuteter oder manifester Sepsis als Tracer-Diagnose regelmäßig hinsichtlich der Versorgungsqualität, -zeiten, Voranmeldung und Übergabe an Schnittstellen ausgewertet und gegebenenfalls optimiert werden.
Fazit für die Praxis

- Die Sepsis ist ein häufiges, vital bedrohliches und oft fulminant verlaufendes Krankheitsbild.

- Jeder Verdacht soll ausgesprochen und differenzialdiagnostisch berücksichtigt werden.

- Die Therapie ist zeitkritisch und folgt dem Prinzip der "golden hour".

- Prähospitale Schwerpunkte:

- Sorgfältige Anamnese und Untersuchung.

- Stabilisierung der Vitalparameter nach „ABCDE-Schema“.

- Falls indiziert Notfallnarkose, Atemwegssicherung und lungenprotektive Beatmung.

- Volumentherapie mit balancierter Vollelektrolytlösung $(30 \mathrm{ml} / \mathrm{kgKG}$ in $3 \mathrm{~h}$ ) nach Blutdruck (MAP $\geq 65 \mathrm{~mm} \mathrm{Hg}$ bzw. systolisch $\geq 100 \mathrm{~mm} \mathrm{Hg}$; primär) und Rekapillarisierungszeit $(<3 \mathrm{~s})$ bzw. Laktat ( $<2 \mathrm{mmol} /$; sekundär) gesteuert.

- Häufig sind zur hämodynamischen Stabilisierung zusätzlich Katecholamine erforderlich.

- Die Auswahl einer geeigneten Klinik und Voranmeldung soll weitere Behandlungspfade triggern und eine gezielte Therapie sowie schnellstmögliche Fokussanierung ermöglichen.

- Die Gewinnung mikrobiologischer Proben und Einleitung der antiinfektiven Therapie sind prähospital prinzipiell machbar, wenngleich diese nicht flächendeckend vorgehalten werden und die Evidenzlage limitiert ist.

- Wie bei allen häufigen und bedrohlichen Notfällen ist regelmäßiges Training wichtig.

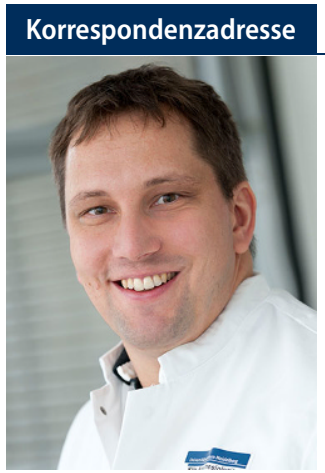

Dr. Manuel Obermaier

Klinik für Anästhesiologie, Universitätsklinikum Heidelberg

Im Neuenheimer Feld 420, 69120 Heidelberg, Deutschland

manuel.obermaier@med.uni-heidelberg.de 
Funding. Open Access funding enabled and organized by Projekt DEAL.

\section{Einhaltung ethischer Richtlinien}

Interessenkonflikt. M. Obermaier, M.A. Weigand, E. Popp und F. Uhle geben an, dass kein Interessenkonflikt besteht.

Für diesen Beitrag wurden von den Autoren keine Studien an Menschen oder Tieren durchgeführt. Für die aufgeführten Studien gelten die jeweils dort angegebenen ethischen Richtlinien.

Open Access. Dieser Artikel wird unter der Creative Commons Namensnennung 4.0 International Lizenz veröffentlicht, welche die Nutzung, Vervielfältigung, Bearbeitung, Verbreitung und Wiedergabe in jeglichem Medium und Format erlaubt, sofern Sie den/die ursprünglichen Autor(en) und die Quelle ordnungsgemäß nennen, einen Link zur Creative Commons Lizenz beifügen und angeben, ob Änderungen vorgenommen wurden.

Die in diesem Artikel enthaltenen Bilder und sonstiges Drittmaterial unterliegen ebenfalls der genannten Creative Commons Lizenz, sofern sich aus der Abbildungslegende nichts anderes ergibt. Sofern das betreffende Material nicht unter der genannten Creative Commons Lizenz steht und die betreffende Handlung nicht nach gesetzlichen Vorschriften erlaubt ist, ist für die oben aufgeführten Weiterverwendungen des Materials die Einwilligung des jeweiligen Rechteinhabers einzuholen.

Weitere Details zur Lizenz entnehmen Sie bitte der Lizenzinformation auf http://creativecommons.org/ licenses/by/4.0/deed.de.

\section{Literatur}

1. Abdullah S, Grand J, Sijapati A et al (2020) qSOFA is a useful prognostic factor for 30-day mortality in infected patients fulfilling the SIRS criteria for sepsis. Am J Emerg Med 38:512-516

2. Ait-Oufella H, Lemoinne S, Boelle PY et al (2011) Mottling score predicts survival in septic shock. Intensive Care Med 37:801-807

3. Alam N, Oskam E, Stassen PM et al (2018) Prehospital antibiotics in the ambulance for sepsis: a multicentre, open label, randomised trial. Lancet RespirMed 6:40-50

4. Barz U, Krahl S, Behringer T et al (2018) Handlungsempfehlungen für Notfallsanitäterinnen und Notfallsanitäter in Baden-Württemberg. Ministerium für Soziales und Integration BadenWürttemberg, Stuttgart

5. Bayer O, Schwarzkopf D, Stumme C et al (2015) An early warning scoring system to identify septic patients in the prehospital setting: the PRESEP score. Acad Emerg Med 22:868-871

6. Bernhard M, Bein B, Böttiger BW et al (2015) Handlungsempfehlung zur prähospitalen Notfallnarkose beim Erwachsenen. Notfall Rettungsmed 18:395-412

7. Bodmann K-F, Grabein BA, Kresken $M$ et al (2019) S2k-Leitlinie: Kalkulierte parenterale Initialtherapie bakterieller Erkrankungen bei Erwachsenen - Update 2018 (AWMF 082/006)

8. Bone RC, Balk RA, Cerra FB et al (1992) Definitions for sepsis and organ failure and guidelines for the

\section{Sepsis in out-of-hospital emergency medicine. What can prehospital emergency care providers derive from the $\mathrm{S} 3$ guideline?}

Background: Sepsis is a challenge in emergency medicine, as this life-threatening organ dysfunction, caused by a dysregulated host response to an infection, presents manifold and therefore is often recognized too late.

Objectives: Recently published surviving sepsis campaign and German S3 guidelines provide recommendations for diagnosis and therapy of sepsis in an in-hospital or intensive care setting, but do not particularly address out-of-hospital emergency medical care. We aim to work out the evidence base with regard to the out-of-hospital care of patients with suspected sepsis and to derive treatment recommendations for emergency medical services.

Conclusions: Therapy of sepsis and septic shock is summarized in bundles, whereby the first bundle should ideally be completed within the first hour-in analogy to "golden hour" concepts in other emergency medical entities, such as trauma care. In the outof-hospital setting, therapy focuses on securing vital parameters, according to the $A B C D E$ scheme, with a particular focus on volume therapy. Further procedures within the $1 \mathrm{~h}$ bundle, such as lactate measurement, obtaining microbiological samples, and starting an anti-infective therapy, are broadly available in hospital only. The aim is to control the site of infection as soon as possible. Therefore, an appropriate designated hospital should be chosen carefully and informed in advance, in order to initiate and pave the way for further clinical diagnostic and treatment paths. Moreover, structured and target-oriented handovers, as well as regular training, are required.

\section{Keywords}

Focus · Septic shock · Infections · Emergency medical services · Antiinfectives

use of innovative therapies in sepsis. The ACCP/ SCCM consensus conference committee. American college of chest physicians/society of critical care medicine. Chest 101:1644-1655

9. Brunkhorst FM, Weigand MA, Pletz $M$ et al (2018) S3-Leitlinie: Sepsis - Prävention, Diagnose, Therapie und Nachsorge (AWMF 079/001)

10. Casu S, Blau J, Schempf B et al (2018) If you don't take a temperature, you can't find a fever. Notfall Rettungsmed 22:509-513

11. Casu S, Haske D (2016) Severe sepsis and septic shock in pre-hospital emergency medicine: survey results of medical directors of emergency medical services concerning antibiotics, blood cultures and algorithms. Intern Emerg Med 11:571-576

12. Chaudhary T, Hohenstein C, Bayer O (2014) Die goldene Stunde der Sepsis: Frühzeitiger präklinischer Therapiebeginn. Med Klin Intensivmed Notfmed 109:104-108

13. Diehlmann F (2015) Erregerdiagnostik und antibiotische Therapie bei antibiotisch nicht vorbehandelten Sepsis-Patienten im Rahmen der IMPACT Sepsis Studie. Julius-MaximiliansUniversität, Würzburg, S117

14. Engel C, Brunkhorst FM, Bone HG et al (2007) Epidemiology of sepsis in Germany: results from a national prospective multicenter study. Intensive Care Med 33:606-618

15. Evans L, Rhodes A, Alhazzani W et al (2021) Surviving sepsis campaign: international guidelines for management of sepsis and septic shoc 2021. Intensive Care Med online (ahead of print). https:// doi.org/10.1007/s00134-021-06506-y

16. Fischer M, Kehrberger E, Marung $\mathrm{H}$ et al (2016) Eckpunktepapier 2016 zur notfallmedizinischen Versorgung der Bevölkerung in der Prähospitalphase und in der Klinik. Notfall Rettungsmed 19:387-395
17. Freund Y, Khoury A, Mockel Metal (2019) European society of emergency medicine position paper on the 1-hour sepsis bundle of the surviving sepsis campaign: expression of concern. Eur JEmerg Med 26:232-233

18. Hernandez G, Ospina-Tascon GA, Damiani LP et al (2019) Effect of a resuscitation strategy targeting peripheral perfusion status vs serum lactate levels on 28-day mortality among patients with septic shock: the ANDROMEDA-SHOCK randomized clinical trial. JAMA 321:654-664

19. Kaukonen KM, Bailey M, Pilcher D et al (2015) Systemic inflammatory response syndrome criteria in defining severe sepsis. N Engl J Med 372:1629-1638

20. Klee Y (2016) Bedeutung des primären Infektfokus für die Morbidität und Mortalität bei Patienten mit Sepsis. Georg-August-Universität, Göttingen, S61

21. Kumar A, Roberts D, Wood KE et al (2006) Duration of hypotension before initiation of effective antimicrobial therapy is the critical determinant of survival in human septic shock. Crit Care Med 34:1589-1596

22. Levy MM, Fink MP, Marshall JC et al (2003) 2001 SCCM/ESICM/ACCP/ATS/SIS international sepsis definitions conference. Crit Care Med 31:1250-1256

23. Long B, Koyfman A (2017) Clinical mimics: an emergency medicine-focused review of sepsis mimics. J Emerg Med 52:34-42

24. Long B, Koyfman A, Modisett KL et al (2017) Practical considerations in sepsis resuscitation. JEmerg Med 52:472-483

25. Marx G, Weigand MA (2017) Sepsis-3: Zweifel an der Überlegenheit der neuen Definition. Dtsch Arztebl 114:1429

26. Mellhammar L, Linder A, Tverring J et al (2019) NEWS2 is superior to qSOFA in detecting sepsis with 
organ dysfunction in the emergency department. JClin Med 8:1128

27. Oda S, Hirasawa H, Sugai T et al (2000) Comparison of sepsis-related organ failure assessment (SOFA) score and CIS (cellular injury score) for scoring of severity for patients with multiple organ dysfunction syndrome (MODS). Intensive Care Med 26:1786-1793

28. Peltan ID, Mitchell KH, Rudd KE et al (2018) Prehospital care and emergency department door-to-antibiotic time in sepsis. Ann Am Thorac Soc 15:1443-1450

29. Polito CC, Isakov A, Yancey AH 2nd et al (2015) Prehospital recognition of severe sepsis: development and validation of a novel EMS screening tool. Am J Emerg Med 33:1119-1125

30. Prasad PA, Fang MC, Abe-Jones $Y$ et al (2020) Time to recognition of sepsis in the emergency department using electronic health record data: a comparative analysis of systemic inflammatory response syndrome, sequential organ failure assessment, and quick sequential organ failure assessment. Crit Care Med 48:200-209

31. Deutscher Berufsverband Rettungsdienst (2020) Muster-Algorithmen 2021 zur Umsetzung des Pyramidenprozesses im Rahmen des NotSanG. Deutscher Berufsverband Rettungsdienst (DBRD), Lübeck

32. Rhee C, Chiotos K, Cosgrove SE et al (2021) Infectious diseases society of america position paper: recommended revisions to the national severe sepsis and septic shock early management bundle (SEP-1) sepsis quality measure. Clin Infect Dis 72:541-552

33. Richter DC, Heininger A, Brenner T et al (2017) Bacterial sepsis: diagnostics and calculated antibiotic therapy. Anaesthesist 66:737-761

34. Royal College of Physicians (2017) National early warning score (NEWS) 2: standardising the assessment of acute-illness severity in the NHS Royal College of Physicians, London

35. Rudd KE, Johnson SC, Agesa KM et al (2020) Global, regional, and national sepsis incidence and mortality, 1990-2017: analysis for the global burden of disease study. Lancet 395:200-211

36. Scheer CS, Fuchs C, Grundling M et al (2019) Impact of antibiotic administration on blood culture positivity at the beginning of sepsis: a prospective clinical cohort study. Clin Microbiol Infect 25:326-331

37. Schmoch $T$, Bernhard $M$, Siegler $B H$ et al (2018) Hämodynamische Stabilisierung des septischen Patienten in der Notaufnahme. Notfall Rettungsmed 22:205-218

38. Schneider K, Bayer O, Schwarzkopf D et al (2015) Sepsis im Rettungsdienst: Effekte einer präklinisch eingeleiteten Sepsistherapie. Anasth Intensivmed 56:PO-2.1.12

39. Sepnet Critical Care Trials Group (2016) Incidence of severe sepsis and septic shock in German intensive care units: the prospective, multicentre INSEP study. Intensive Care Med 42:1980-1989

40. Seymour CW, Gesten F, Prescott HC et al (2017) Time to treatment and mortality during mandated emergency care for sepsis. N Engl J Med 376:2235-2244

41. Seymour CW, Rea TD, Kahn JM et al (2012) Severe sepsis in pre-hospital emergency care: analysis of incidence, care, and outcome. Am J Respir Crit Care Med 186:1264-1271

42. Shapiro NI, Wolfe RE, Moore RB et al (2003) Mortality in emergency department sepsis (MEDS) score: a prospectively derived and validated clinical prediction rule. Crit Care Med 31:670-675
43. Singer M, Deutschman CS, Seymour CWetal (2016) The third international consensus definitions for sepsis and septic shock (sepsis-3). JAMA 315:801-810

44. Ständige Impfkommission (2019) Empfehlungen der Ständigen Impfkommission (STIKO) am Robert-Koch-Institut. Epidemiol Bull 34:313-364

45. Timmermann A, Böttiger BW, Byhahn C et al (2019) S1-Leitlinie: Prähospitales Atemwegsmanagement. Anasth Intensivmed 60:316-336

46. Tusgul S, Carron PN, Yersin B et al (2017) Low sensitivity of qSOFA, SIRS criteria and sepsis definition to identify infected patients at risk of complication in the prehospital setting and at the emergency department triage. Scand J Trauma Resusc Emerg Med 25:108

47. Umscheid CA, Betesh J, Vanzandbergen C et al (2015) Development, implementation, and impact of an automated early warning and response system for sepsis. J Hosp Med 10:26-31

48. Vincent JL, Moreno R, Takala J et al (1996) The SOFA (sepsis-related organ failure assessment) score to describe organ dysfunction/failure. On behalf of the working group on sepsis-related problems of the European society of intensive care medicine. Intensive Care Med 22:707-710

49. Walchok JG, Pirrallo RG, Furmanek D et al (2017) Paramedic-initiated CMS sepsis core measure bundle prior to hospital arrival: a stepwise approach. Prehosp Emerg Care 21:291-300
Burger | Egger | Heschl | Huber | Isser Pimiskern | Rauter | Schiefer Erste Hilfe in den Bergen. Unfälle und Notfälle beim Wandern, Bergsteigen und Klettern.

Springer Berlin, Heidelberg 2021, 171 S., 86 Abb., (ISBN: 978-3-66262497-5), 19,90 EUR

Die Autoren des Buches sind erfahrenen Bergrettungsärzten und Bergrettern aus Österreich. Das komplette Spektrum der Verletzungen und Erkrankungen, die im alpinen Gelände bei Bergsteigern, Wanderern oder Klettern auftreten können, wird abgedeckt: vom Sonnenstich, über den Schlangenbiss bis hin zu schweren Verletzungen und dem Lawinenunfall angereichert durch praktische Tipps zum Packen der Rucksackapotheke, und Tricks beispielsweise zum Schienen von Fingerverletzungen, zum Schienen des Unterarms mit der Jake des Patienten oder dem Entfernen von Ringen an den Fingern nach Handverletzung. Das Buch liest sich trotz der Vielzahl der Autoren „wie aus einem Guss" ohne unnötige Wiederholungen.

\section{Zielpublikum des Buches}

Das Buch umfasst - wie der Titel sagt - nur die „Erste Hilfe". Es behandelt also nicht die Versorgung durch den professionellen (Berg-)Rettungsdienst, vielmehr ist es auf interessierte Bergsportler, die sich optimal auf mögliche medizinische Notfälle im alpinen Gelände vorbereiten will, ausgerichtet. Ideal geeignet ist das Buch sicher auch für die medizinische Ausbildung angehender Hochtourenführer oder Wanderleiter. Medizinisches Fachpersonal kann das Buch auch nutzen, um sich grob orientierend auf Bergmedizinkurse vorzubereiten. Für ein Bestehen der Kurse reicht jedoch die Tiefe der behandelten Themen allein nicht aus. Dennoch fand ich persönlich - selbst als langjährig erfahrener Arzt mit viel Erfahrung im alpinen Gelände - die Lektüre kurzweilig, interessant und insbesondere die gezeigten „Tricks" zur behelfsmäßigen Schienung von Frakturen haben mich beeindruckt und zeugen von der umfassenden Erfahrung des Autorenteams.

Priv.-Doz. Dr. Jürgen Knapp, Bern 